\title{
BMJ Open Identification of risk factors affecting catch-up growth after infant congenital heart disease surgery: rationale and design of a multicentre prospective cohort study in China
}

\author{
Lijuan Li, ${ }^{1}$ Kuanrong LI, ${ }^{2}$ Caixia An, ${ }^{3}$ Jiajie Fan, ${ }^{4}$ Changying Guo, ${ }^{5}$ Suixin Liang, ${ }^{6}$ \\ Yue Guo, ${ }^{3}$ Huimin Xia, ${ }^{7}$ Xinxin Chen, ${ }^{1}$ Yanna Zhu, ${ }^{8}$ Chunmei Hu, ${ }^{1}$ Wenyue $\mathrm{Si}^{2}{ }^{2}$ \\ Huiying Liang, ${ }^{2}$ Yanqin Cui $^{1}$
}

To cite: Li L, LI K, An C, et al. Identification of risk factors affecting catch-up growth after infant congenital heart disease surgery: rationale and design of a multicentre prospective cohort study in China. BMJ Open 2019;9:e030084. doi:10.1136/ bmjopen-2019-030084

- Prepublication history and additional material for this paper are available online. To view these files, please visit the journal online (http://dx.doi. org/10.113/6bmjopen-2019030084).

$\mathrm{LL}$ and $\mathrm{KL}$ contributed equally to this work.

Received 26 February 2019 Revised 09 July 2019 Accepted 12 July 2019

Check for updates

(C) Author(s) (or their employer(s)) 2019. Re-use permitted under CC BY-NC. No commercial re-use. See rights and permissions. Published by BMJ.

For numbered affiliations see end of article.

Correspondence to

Dr Yanqin Cui;

cuiyanqin@gwcmc.org

\section{ABSTRACT}

Introduction Successful surgical treatment of congenital heart disease improves neonates' long-term survival and leads to catch-up growth, which however does not occur in part of the patient population for largely undetermined reasons.

Methods and analysis A multicentre, prospective cohort study is being conducted in four paediatric medical institutions in China to collect detailed nutritional, anthropometric and clinical data at perioperative phases and during a 1-year period of follow-up after surgery. The study is expected to recruit approximately 5000 patients by the year of 2023 when the cohort is fully established. The primary endpoint of this study is the occurrence of postoperative catch-up growth, which will be determined in both absolute and relative terms (ie, reduced anthropometric deficits from the reference measures and improved z-scores that have passed the -2 SD cut-offs). Multivariable regression analyses will be performed to identify factors that are statistically significantly associated with the absence of postoperative catch-up growth.

Ethics and dissemination The protocol of this study has been approved by the individual ethics committees of the participating centres (Guangzhou Women and Children's Medical Centre (2008071601), the Children's Hospital of Zhejiang University School of Medicine (2018-IRB094), Gansu Provincial Maternity and Child-Care Hospital (2019-IRB-01) and Zhengzhou Cardiovascular Hospital (2019012001)). Written informed consent from parents will be obtained before study entry. Findings of this study will be disseminated through publications in international peer-reviewed journals and will be presented in academic conferences.

\section{INTRODUCTION}

Congenital heart disease (CHD) accounts for nearly one-third of all major congenital anomalies combined and affects more than 1 million neonates each year globally. ${ }^{1}$ Owing to CHD-associated pathophysiological changes such as low energy intake,

\section{Strengths and limitations of this study}

- This is the first prospective cohort study aiming to reveal the risk factors for lack of catch-up growth despite successful surgical repair of cardiac anomalies during infancy.

- The strengths of this study include its prospective design, large cohort size (expectedly 5000 patients) and comprehensive collection of data.

- Selection bias and potentially high loss-to-follow-up rate are the limitations of the study.

malabsorption, hypermetabolism and sometimes coexisting gastrointestinal malformations, neonates with CHD are at high risk of developing malnutrition during infancy. ${ }^{2-7}$ Previous studies have reported that almost half of infants with CHD are stunted and 15\% of the patients suffer from moderate-to-severe malnutrition. ${ }^{89}$

In addition to significantly improving the long-term survival of neonates, successful surgical treatment of CHD may also deliver an accelerated growth rate, which usually is referred to as catch-up growth. ${ }^{410-17}$ However, studies have shown that postoperative catch-up growth was less pronounced or even absent in $15 \%-50 \%$ of patients depending on the study. ${ }^{4215}$ Failure to observe catch-up growth is presumed to be multidimensional and involving multiple patient-related and surgery-related factors but so far remains largely uninvestigated with only two studies where severe malnutrition before surgery, parental height and developmental delay were identified as potentially associated. ${ }^{413} 18$

In this article, we report the design and rationale of a multicentre, prospective cohort study that aims to identify perioperative 
factors that are associated with the likelihood of successful or unrealised catch-up growth after surgery for major congenital heart defects and to provide insights into the possible mechanisms.

\section{METHODS AND ANALYSIS}

\section{Patients and public involvement}

Neither patients nor the public were involved in the design of this study. The findings of this study will be available to the patients and the public once they have been published.

\section{Study design and patient recruitment}

This is a prospective, multicentre cohort study being carried out at four tertiary medical institutions situated in four provinces across China (see online supplementary figure), that is, Guangzhou Women and Children's Medical Center in Guangzhou (GZ, Guangdong Province), the Children's Hospital of Zhejiang University School of Medicine in Hangzhou (HZ, Zhejiang Province), Gansu Provincial Maternity and Child-Care Hospital in Lanzhou (LZ, Gansu Province) and Zhengzhou Cardiovascular Hospital in Zhengzhou (ZZ, Henan Province). All the study centres are tertiary referral hospitals but located in regions with different cultural and socioeconomic characteristics. Selection of the four study centres was mainly based on the standardised surgical treatment of infant CHD across these centres and their representativeness of patients with different socioeconomic backgrounds.

Patient recruitment for this study commenced in September 2018 and will continue until the year of 2023. The study population from each centre consists of consecutive infants ( $<12$ months of age) who are admitted with CHD and who will undergo surgical correction. Patients with any of the following characteristics are excluded: (1) have any diagnosed chromosomal diseases (eg, Down syndrome); (2) have any diagnosed metabolic diseases (eg, galactosaemia and hypothyroidism); (3) have a history of gastrointestinal surgery; (4) have conditions that restrict oral feeding (eg, cleft palate); (5) have parents who are illiterate.

\section{Follow-up protocol}

After CHD surgery, all study participants will be followed for 1 year, during which they are invited to return to the medical centre for detailed medical examinations and anthropometric measurements. These evaluations will take place at 1, 3, 6 and 12 months after surgery. In order to maintain a high follow-up rate, study participants will be contacted regularly by phone and will stay informed of the study's progress by receiving newsletters from the study centres.

\section{Data collection}

Table 1 lists the clinical examinations and assessments to be conducted perioperatively and during follow-up. Prior to CHD surgery, detailed information on medical history including CHD-related treatments and medications will be collected. Baseline anthropometric measurements including body weight, recumbent length, head circumference and triceps skin fold will be obtained by trained nurses 1 day before surgery following standardised procedures. Congenital cardiac malformations will be coded using both the International Classification for Diseases 10 th version (ICD-10) and the nomenclature adopted by the Society of Thoracic Surgeons (STS) Congenital Heart Surgery Database. ${ }^{19}$ Likewise, the surgical procedures will be coded using both the ICD ninth verision for Clinical

Table 1 Scheduled examinations

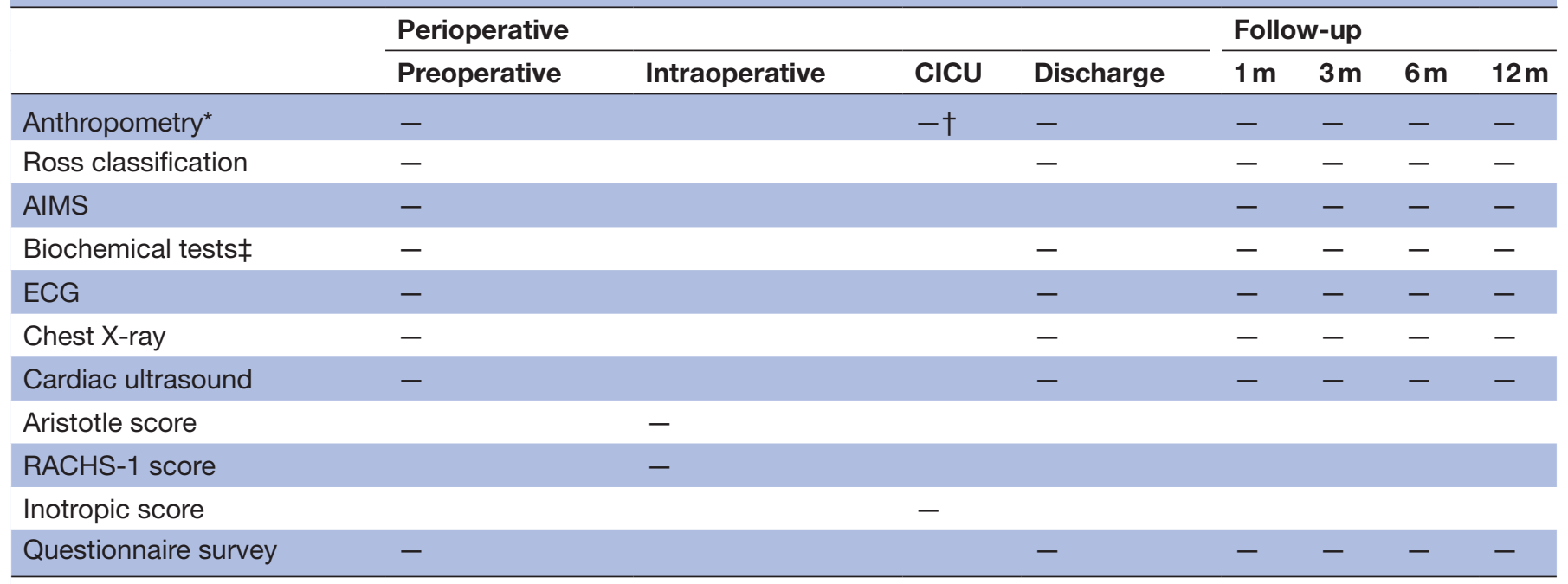

*Including body weight, recumbent length, head circumference, upper arm circumference and triceps skin fold.

†Performed to children with cardiac intenstive care unit (CICU) stay $\geq 5$ days.

$\ddagger$ Complete blood count and blood chemistry tests.

AIMS, Alberta Infant Motor Scale; RACHS, risk adjustment in congenital heart surgery. 
Modification and the STS procedural codes. Grading of the severity of heart failure signs and symptoms will be assessed using the Ross classification method. ${ }^{20}$ The Alberta Infant Motor Scale ${ }^{21}$ will be used to measure the patients' motor development and will be obtained by paediatric rehabilitation technicians who have been trained on paediatric neurology. Biochemical tests on blood samples, ECG and echocardiogram will also be performed. Self-administered questionnaires will be used to collect information on demographics, socioeconomics, mother's reproductive history, gestational vitamin and mineral supplementation, and feeding mode before and after surgery.

\section{Determination of outcomes}

The primary outcomes of the present study are the catch-up growth for weight and height after CHD surgery. Catch-up growth can be defined as growth velocity that is above the average level for the child's age and sex after a period of retardation. ${ }^{1022} 23$ According to this definition, a child who used to have a weight-for-age z-score or height-for-age z-score more than 2 SD below the mean of the healthy reference population is classified as having catch-up growth if he/she has shown an improved z-score that is above the -2 SD cut-off. Besides the improved z-scores, which are relative measures of catch-up growth, we will also consider the absolute decreases in anthropometric deficits, given the fact that absolute anthropometric deficits might become more marked despite improved z-scores because of the continuously increasing SD during childhood. ${ }^{24} 25$

In addition to the anthropometric outcomes, this study will also investigate the fluctuations of important biomedical indicators of malnutrition before and after CHD surgery, including but not limited to albumin, prealbumin, retinol-binding protein, transferrin and N-terminal probrain natriuretic peptide.

\section{Sample size}

The annual number of infant CHD surgery performed in each study centre is about 500 in GZ, 300 in HZ and 100 in each of ZZ and LZ; therefore, this study is expected to recruit around 5000 patients during the recruitment period (2018-2023). As this study is a primary epidemiological investigation without any predefined statistical hypothesis to test, a priori sample size calculation is unnecessary. ${ }^{26}$

\section{Statistical analyses}

Perioperative characteristics of the study participants will be described with either arithmetic mean or percentages. The incidence rates of catch-up growth between two adjacent time points and during the entire period of follow-up will be determined. Analysis of variance and $\chi^{2}$ test for repeated measures will be used to test for statistically significant differences in continuous and categorical outcomes, respectively.

Patients' z-cores will be calculated using WHO growth charts as the reference. Multivariable Cox regression models will be fitted to identify risk factors associated with the absence of postoperative catch-up growth, with age as the time scale extending from CHD surgery until the presence of catch-up growth, censoring (death/ withdrawal/loss of contact), or the end of the follow-up, whichever comes first. Table 2 shows the factors that we consider might have associations with postoperative catch-up growth and therefore will be examined in the present study. Random effects will be modelled to deal with the fact that patients in the same study centre are likely to share some common characteristics. The models will be extended to handle time-varying factors (eg, laboratory results).

\section{DISCUSSION}

Malnutrition is prevalent among paediatric patients with CHD. ${ }^{41527-29}$ Energy metabolism plays a key role in infant growth and disturbed metabolic balance leads to deviation from the normal growth trajectory. Studies have also shown that, for children with CHD, their total and resting energy expenditures normalise in a few weeks

Table 2 Factors that will be considered in risk association analysis for postoperative catch-up growth

\begin{tabular}{ll}
\hline Patient features & $\begin{array}{l}\text { Demographics: age, sex, birth order; parent socioeconomic status; gestational age; birth weight and length; intrauterine } \\
\text { factors; breast feeding; timing of CHD diagnosis (early: antenatal or predischarge from birth admission; late: postdischarge } \\
\text { from birth admission) }\end{array}$ \\
Preoperative & $\begin{array}{l}\text { Anthropometric measurements; nutrition support (route, timing, caloric intake and length); formula type; gastrointestinal } \\
\text { infection; mechanical ventilation; AIMS; Ross score; non-cardiac congenital abnormality; laboratory results (haemoglobin, } \\
\text { total serum protein, albumin, prealbumin, transferrin, retinol-binding protein, C reactive protein); cardiac function (Ross score, } \\
\text { echocardiogram, NT-proBNP) }\end{array}$ \\
Surgery & $\begin{array}{l}\text { Type of surgery; RACHS-1 score; Aristotle score } \\
\text { CICU }\end{array}$ \\
$\begin{array}{l}\text { Nutrition support (route, timing, caloric intake and length); target energy intake; micronutrient supplementation; postoperative } \\
\text { nosocomial infection; residual lesion and timing of reintervention; inotropic score, length of mechanical ventilation; length of }\end{array}$ \\
CICU stay.
\end{tabular}

AIMS, Alberta Infant Motor Scale; CHD, congenital heart disease; CICU, cardiac intensive care unit; NT-proBNP, N-terminal probrain natriuretic peptide; RACHS, risk adjustment in congenital heart surgery. 
after successful repair of the cardiac lesions, resulting in prompt improvement in growth. ${ }^{30-32}$

However, this 'catch-up growth' following CHD surgery only occurs in some patients ${ }^{41833}$ and determinants of catch-up growth have not been established in currently available studies. ${ }^{41416}$ Early surgery was thought to be associated with a better catch-up growth, but existing evidence is conflicting. ${ }^{31-1432}$ Low parental height was found to be associated with a risk of postoperative malnutrition but only in one study. ${ }^{4}$

Many other perioperative factors might also influence postoperative nutritional status and hence deserve detailed examination. Feeding routes (tube vs oral) have been reported to be associated with preoperative nutritional status. ${ }^{34}$ One study identified hypoplastic left heart syndrome, longer intubation and duration of withholding enteral feeds, and gastro-oesophageal reflux as risk factors for poor feeding among infants with CHD. ${ }^{35}$ In another study, the extent of growth retardation was greater in cyanotic than in acyanotic patients, while the greatest improvement in growth occurred in patients with isolated valvular pulmonary stenosis, ${ }^{36}$ suggesting that nutritional outcomes after surgical intervention might vary by underlying cardiac malformations.

To the best of our knowledge, the present study is the first prospective cohort designed for a thorough investigation of perioperative factors that are associated with persistent growth deficits after infant CHD surgery. This study will provide a good opportunity to assess a wide range of perioperative predictors that have never been examined before. The multicentre design will contribute to a more heterogeneous study population, which improves the generalisability of the results. The prospective nature of this study will help reduce selection bias and improve data quality, compared with retrospective studies. The present study has several limitations including a relatively short duration of follow-up and potentially high rate of loss to follow-up. One year follow-up may limit the assessment of catch-up growth in height, which can require more than 1 year. Because all the participating centres are tertiary referral hospitals, it is likely that a considerable number of study participants do not live nearby the study centres, and some of them will be unwilling to travel a long way back for follow-up examinations.

In summary, given that lack of catch-up growth is relatively common among infants after surgical repair of congenital cardiac anomalies and contributing factors have not been well elucidated, we believe that this prospective study will yield useful information for risk stratification and to identify potential causal mechanisms.

\section{Author affiliations}

${ }^{1} \mathrm{CICU}$, Guangzhou Women and Children's Medical Center, Guangzhou Medical University, Guangzhou, China

'Institute of Paediatrics, Guangzhou Women and Children's Medical Center, Guangzhou, China

${ }^{3} \mathrm{CICU}$, Gansu Provincial Maternity and Child-Care Hospital, Lanzhou, China ${ }^{4} \mathrm{CICU}$, The Children's Hospital of Zhejiang University School of Medicine, Hangzhou, China
${ }^{5} \mathrm{CICU}$, Zhengzhou Cardiovascular Hospital, Zhengzhou, China

${ }^{6} \mathrm{CICU}$, Shenzhen Children's Hospital, Shenzhen, China

${ }^{7}$ Guangdong Key Laboratory of Structural Birth Defects, Guangzhou, China

${ }^{8}$ Department of Maternal and Child Health, Sun Yat-sen University School of Public Health, Guangzhou, China

Acknowledgements The authors are thankful to the patients who have participated in the study and to the research nurses for their contribution to patient recruitment. They also thank Abraham Morse for his help on language editing.

Contributors $\mathrm{LL}$ and $\mathrm{KL}$ drafted the manuscript. YZ developed the baseline and follow-up questionnaires. WS contributed to data management and the design of the follow-up protocol. YC, CA, JF, CG, SL, YG, HX, XC, CH and HL contributed to the conception of the study. YC, CA, JF and CG initiated the study in their study centre. All authors reviewed, read and approved the final manuscript.

Funding This study is funded by the internal grant from the Guangdong Key Laboratory of Structural Birth Defects and the grant from the Guangzhou Municipal Health Council (20191A011024).

Competing interests None declared.

Patient consent for publication Parental/guardian consent obtained.

Ethics approval An ethics approval has been obtained from the ethics committee in each of the four study centres (GZ: 2008071601, HZ: 2018-IRB-094, ZZ: 2019012001, LZ: 2019-IRB-01).

Provenance and peer review Not commissioned; externally peer reviewed.

Open access This is an open access article distributed in accordance with the Creative Commons Attribution Non Commercial (CC BY-NC 4.0) license, which permits others to distribute, remix, adapt, build upon this work non-commercially, and license their derivative works on different terms, provided the original work is properly cited, appropriate credit is given, any changes made indicated, and the use is non-commercial. See: http://creativecommons.org/licenses/by-nc/4.0/.

\section{REFERENCES}

1. van der Linde D, Konings EEM, Slager MA, et al. Birth prevalence of congenital heart disease worldwide: a systematic review and metaanalysis. J Am Coll Cardiol 2011;58:2241-7.

2. Medoff-Cooper B, Ravishankar C. Nutrition and growth in congenital heart disease: a challenge in children. Curr Opin Cardiol 2013;28:122-9.

3. Forchielli ML, McColl R, Walker WA, et al. Children with congenital heart disease: a nutrition challenge. Nutr Rev 1994;52:348-53.

4. Vaidyanathan $B$, Radhakrishnan $R$, Sarala DA, et al. What determines nutritional recovery in malnourished children after correction of congenital heart defects? Pediatrics 2009;124:e294-9.

5. Okoromah CAN, Ekure EN, Lesi FEA, et al. Prevalence, profile and predictors of malnutrition in children with congenital heart defects: a case-control observational study. Arch Dis Child 2011;96:354-60.

6. Egbe A, Uppu S, Lee S, et al. Prevalence of associated extracardiac malformations in the congenital heart disease population. Pediatr Cardiol 2014;35:1239-45.

7. Eskedal L, Hagemo P, Eskild A, et al. A population-based study of extra-cardiac anomalies in children with congenital cardiac malformations. Cardiol Young 2004;14:600-7.

8. Thommessen M, Heiberg A, Kase BF. Feeding problems in children with congenital heart disease: the impact on energy intake and growth outcome. Eur J Clin Nutr 1992;46:457-64.

9. Blasquez A, Clouzeau H, Fayon M, et al. Evaluation of nutritional status and support in children with congenital heart disease. Eur $J$ Clin Nutr 2016;70:528-31.

10. Carmona F, Hatanaka LS, Barbieri MA, et al. Catch-Up growth in children after repair of tetralogy of Fallot. Cardiol Young 2012;22:507-13.

11. Swan JW, Weintraub RG, Radley-Smith R, et al. Long-Term growth following neonatal anatomic repair of transposition of the great arteries. Clin Cardiol 1993;16:392-6.

12. Rhee EK, Evangelista JK, Nigrin DJ, et al. Impact of anatomic closure on somatic growth among small, asymptomatic children with secundum atrial septal defect. Am J Cardiol 2000;85:1472-5.

13. Schuurmans FM, Pulles-Heintzberger CF, Gerver WJ, et al. Longterm growth of children with congenital heart disease: a retrospective study. Acta Paediatr 1998;87:1250-5.

14. Cheung MMH, Davis AM, Wilkinson JL, et al. Long term somatic growth after repair of tetralogy of fallot: evidence for restoration of genetic growth potential. Heart 2003;89:1340-3. 
15. Page RE, Deverall PB, Watson DA, et al. Height and weight gain after total correction of fallot's tetralogy. Br Heart J 1978;40:416-20.

16. Weintraub RG, Menahem S. Early surgical closure of a large ventricular septal defect: influence on long-term growth. J Am Coll Cardiol 1991;18:552-8.

17. Gilboa SM, Salemi JL, Nembhard WN, et al. Mortality resulting from congenital heart disease among children and adults in the United States, 1999 to 2006. Circulation 2010;122:2254-63.

18. Feldt RH, Strickler GB, Weidman WH. Growth of children with congenital heart disease. Am J Dis Child 1969;117:281-579.

19. Mavroudis C, Jacobs JP. Congenital heart surgery Nomenclature and database project: overview and minimum dataset. Ann Thorac Surg 2000;69(4 Suppl):2-17.

20. Ross RD. The Ross classification for heart failure in children after 25 years: a review and an age-stratified revision. Pediatr Cardiol 2012;33:1295-300.

21. Piper MC, Pinnell LE, Darrah J, et al. Construction and validation of the Alberta infant motor scale (aims). Can J Public Health 1992;83(Suppl 2):S46-50.

22. Wit J-M, Boersma B. Catch-Up growth: definition, mechanisms, and models. J Pediatr Endocrinol Metab 2002;15(Suppl 5):1229-41.

23. Desmond C, Casale D. Catch-Up growth in stunted children: definitions and predictors. PLoS One 2017;12:e0189135.

24. Lundeen EA, Stein AD, Adair LS, et al. Height-for-age $Z$ scores increase despite increasing height deficits among children in 5 developing countries. Am J Clin Nutr 2014;100:821-5.

25. Leroy JL, Ruel M, Habicht J-P, et al. Using height-for-age differences (had) instead of height-for-age z-scores (HAZ) for the meaningful measurement of population-level catch-up in linear growth in children less than 5 years of age. BMC Pediatr 2015;15:145.

26. Jones SR, Carley S, Harrison M. An introduction to power and sample size estimation. Emerg Med J 2003;20:453-8.
27. Arodiwe I, Chinawa J, Ujunwa F, et al. Nutritional status of congenital heart disease (CHD) patients: Burden and determinant of malnutrition at university of Nigeria teaching hospital Ituku - Ozalla, Enugu. Pak J Med Sci 2015;31:1140-5.

28. Varan B, Tokel K, Yilmaz G. Malnutrition and growth failure in cyanotic and acyanotic congenital heart disease with and without pulmonary hypertension. Arch Dis Child 1999;81:49-52.

29. Nydegger A, Bines JE. Energy metabolism in infants with congenital heart disease. Nutrition 2006;22:697-704.

30. Leitch CA, Karn CA, Ensing GJ, et al. Energy expenditure after surgical repair in children with cyanotic congenital heart disease. $J$ Pediatr 2000;137:381-5.

31. Leitch CA, Karn CA, Peppard RJ, et al. Increased energy expenditure in infants with cyanotic congenital heart disease. $J$ Pediatr 1998;133:755-60.

32. Nydegger A, Walsh A, Penny DJ, et al. Changes in resting energy expenditure in children with congenital heart disease. Eur J Clin Nutr 2009;63:392-7.

33. Rosenthal A, Castaneda A. Growth and development after cardiovascular surgery in infants and children. Prog Cardiovasc Dis 1975;18:27-37.

34. Schwarz SM, Gewitz MH, See CC, et al. Enteral nutrition in infants with congenital heart disease and growth failure. Pediatrics 1990;86:368-73.

35. Indramohan G, Pedigo TP, Rostoker N, et al. Identification of risk factors for poor feeding in infants with congenital heart disease and a novel approach to improve oral feeding. J Pediatr Nurs 2017;35:149-54.

36. Mehrizi A, Drash A. Growth disturbance in congenital heart disease. J Pediatr 1962;61:418-29. 